\title{
Stiff person syndrome and related disorders
}

INSERM

\section{Source}

INSERM. (1999). Orphanet: an online rare disease and orphan drug data base. Stiff person syndrome and related disorders. ORPHA:3198

Stiff-man syndrome (SMS) is a rare neurological disorder comprising fluctuating trunk and limb stiffness, painful muscle spasms, task-specific phobia, an exaggerated startle response, and ankylosing deformities such as fixed lumbar hyperlordosis. 